\title{
MULTIRESOLUTION SIMULATIONS OF PHOTOINJECTORS
}

\author{
D. Mihalcea, C. L. Bohn and B. Terzić \\ Department of Physics, Northern Illinois University, DeKalb, IL 60115, USA
}

\begin{abstract}
We report a successful implementation of a three-dimensional wavelet-based solver for Poisson's equation with Dirichlet boundary conditions, optimized for use in particle-in-cell beam dynamics simulations. We explain how the new algorithm works and the advantages it brings to accelerator simulations. The solver is integrated into a full photoinjector-simulation code (Impact-T), and the code is then benchmarked by comparing its output against that of other codes (verification) and against laboratory measurements (validation). We also simulated the AES/JLab photoinjector using a suite of codes. This activity revealed certain performance limitations and their causes.
\end{abstract}

Keywords: photoinjector, space charge, Poisson solver, wavelets, free-electron laser

PACS: 41.75.-i, 41.20.Cv, 52.59.Sa

\section{INTRODUCTION}

Free Electron Laser (FEL) applications as well as the future International Linear Collider (ILC) require photoinjectors capable of producing high-brightness and low-emittance electron beams $[1,2]$. Computer simulations are the main tool for the design and optimization of such photoinjectors. Emittance growth occurs mainly in the early stages of the acceleration when the beam is still in the proximity of the photocathode and it is primarily due to space charge [3]. Therefore, accurate evaluation of the space-charge effects is a major goal for all particle tracking codes.

Although azimuthal symmetry can greatly decrease the simulation time, it will not generally hold when the emission of the electrons from the photocathode is inhomogeneous or when there are some misalignments of the various photoinjector components $[4,5]$. For these situations it is necessary that the particle tracking codes include truly three-dimensional Poisson solvers in order to account correctly for the space charge effects $[6,7]$. Optimized algorithms and parallel computation can significantly decrease the duration of the simulations and increase the accuracy by using bunches with a larger number of macroparticles. For example, Impact-T [7] uses parallel computation and a fast Fourier transform (FFT) of the shifted Green function to solve accurately the threedimensional Poisson equation with open boundary conditions.

We developed a wavelet-based algorithm for solving the three-dimensional Poisson equation with Dirichlet boundary conditions which can be used within Impact-T [8] The new wavelet-based algorithm is only slightly faster than the standard one used by Impact-T, but it has two key advantages: (1) the distribution of the charged macroparticles can be smoothed in a meaningful way, and (2) the calculated potential can be compressed and efficiently stored at each tracking time step. 


\section{ADVANTAGES OF WAVELETS}

Wavelets are a complete set of two-variable eigenfunctions used to analyze data in both time and frequency domains $[9,10,11]$. In practice the most widely used wavelet transform of an arbitrary function $f(t)$ is the Discrete Wavelet Transform (DWT) defined by the following equation:

$$
W(j, k)=\sum_{i=1}^{n} f(i) 2^{-j / 2} \Psi^{\star}\left(2^{j}-k\right)
$$

where the function $\Psi$ is the mother wavelet function. Similar to the case of Fourier analysis, each wavelet transform admits a unique inverse. Also, fast algorithms (faster than FFTs) are available for both direct and inverse wavelet transforms. The mother wavelet function in Eq. (1) is not unique, but for a large range of practical applications the wavelet families described in [11] are well suited because they have compact support and are continuous.

In the new wavelet-based space-charge algorithm, the Poisson equation is first discretized on a three dimensional grid and then converted to the wavelet space. In this space the Poisson equation is solved iteratively by using a Preconditioned Conjugate Gradient (PCG) operator. Once the solutions are found, they are converted back to the physical space domain. The reason for solving the Poisson equation in a wavelet space is that the speed of the iterative method is greatly increased by a very efficient preconditioning operator [8]. This compensates the extra time spent with the wavelet transforms. As a bonus, this wavelet-based Poisson solver includes two more useful features which come at no extra cost: denoising of the charge distribution and compact storage of the calculated potential.

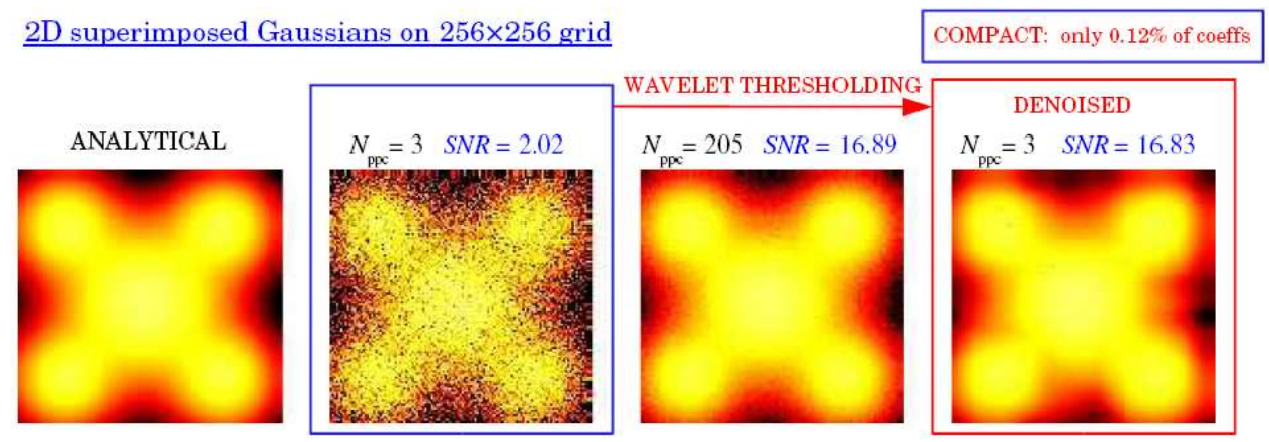

FIGURE 1. Wavelet-based denoising for a two-dimensional distribution when the average number of Particles Per Cell (PPC) is 3 and Signal to Noise Ratio (SNR) is 2.02. SNR can be increased to about 17 by either increasing the PPC to 205 or by thresholding the wavelet transform of the initial distribution. In the latter case there is no increase of the PPC and only a small fraction of the wavelet coefficients are kept.

The wavelet transform of the charge distribution typically contains only a small percentage of terms significantly different from zero. Therefore, it is possible to smooth the charge distribution by setting to zero all terms in the wavelet transform which exceed a certain threshold. This denoising procedure is exemplified in Fig. 1 for a two- 
dimensional case. Since the small terms in the wavelet transform are associated with noise, it is also possible to store the calculated potential in a very efficient way. By keeping only a small percentage of the wavelet coefficients, the potential is only very slightly affected and the memory needed for storage is about 50 times smaller.

\section{CODE VERIFICATION AND VALIDATION}

The wavelet-based code was incorporated in the particle tracking code Impact-T as a distinct package for space charge calculation. To verify the new code we ran Impact$\mathrm{T}$ either with its standard space charge algorithm or with the wavelet-based code and compared the results for beam moments and slices of the phase-space distributions.
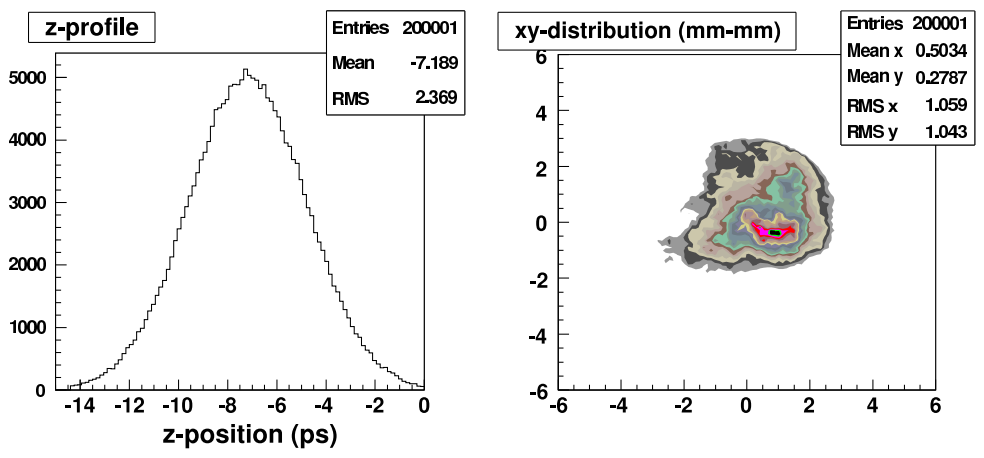

FIGURE 2. Initial space charge distribution: longitudinal (left) and transverse (right).

Since here our primary interest is photoinjectors, the input files of the codes we want to compare include the lattice of the Fermilab/NICADD photoinjector [12]. The initial particle distribution, Fig. 2, was generated to mimic a real distribution with pronounced nonuniformity to make sure that space charge-effects have a big role in particle dynamics.

The results for the most common beam moments are shown in Fig. 3 when the photoinjector operates in standard conditions and the electron bunch charge is $1 \mathrm{nC}$. In the case of Impact- $T$ with the wavelet-based algorithmi, the runs were made with and without denoising. Since the number of macroparticles in the simulations is large $(200,000)$ we didn't see any major effect from denoising. The agreement between the codes is within $5 \%$.

A more detailed verification of the wavelet-based code can be achieved by comparing slices of the phase space at different distances from the cathode. Figure 4 shows transverse particle distributions at several $z$-locations for both space-charge algorithms. In this case, the radial and azimuthal nonuniformity of the space-charge forces are due to the initial 5-beamlet quincunx transverse particle distribution.

To validate the new wavelet-based code we compared the results from the simulations with available experimental data. Figure 5 shows how experimental measurements of the beam radius at different locations down the beamline compare with the simulations. Figure 6 compares the measured transverse emittances of the beam with the results given by the two versions of Impact-T. The emittances were measured at about $4 \mathrm{~m}$ from the 

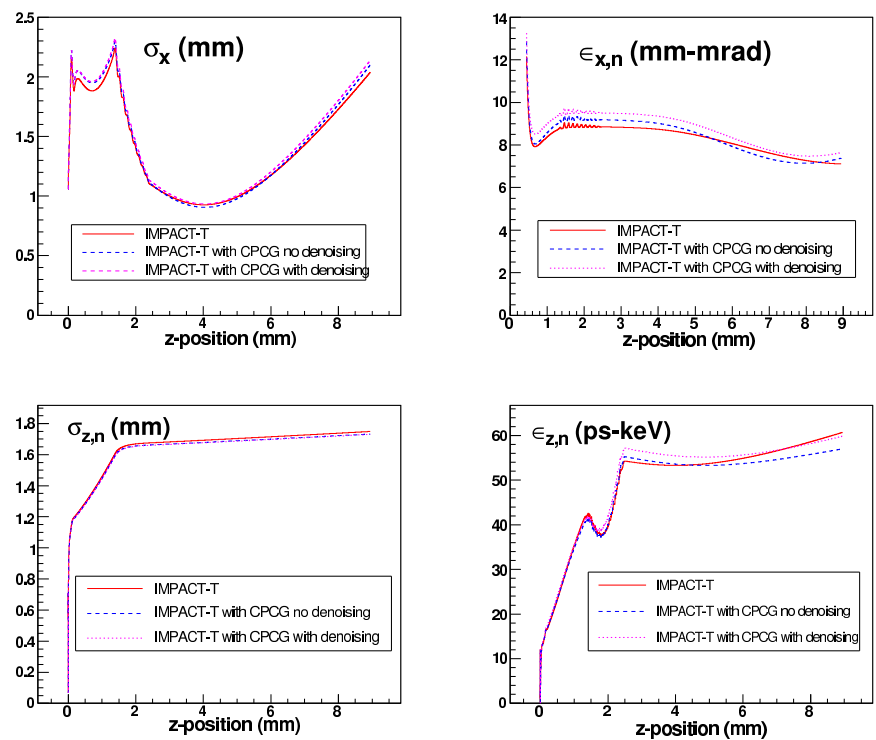

FIGURE 3. Beam moments for a $1 \mathrm{nC}$ beam at Fermilab/NICADD photoinjector.
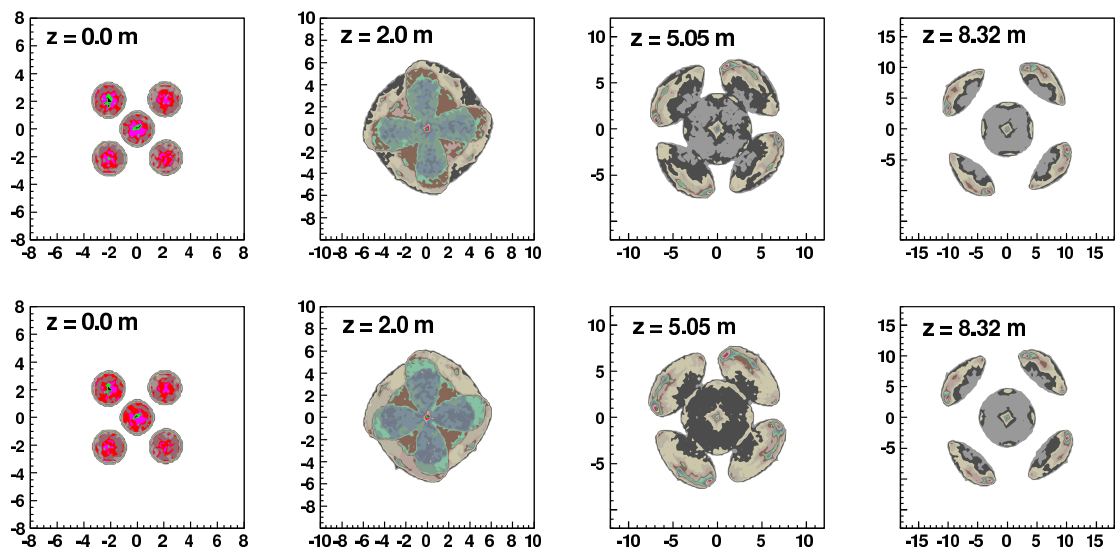

FIGURE 4. Transverse particle distributions at different distances from the cathode: (top) computed with wavelets and denoising; (bottom) computed with standard Impact-T.

cathode as a function of the magnetic field inside the emittance-compensation solenoids. The comparisons shown in Figs. 5 and 6 clearly demonstrate that simulations with both versions of Impact-T are in very good agreement with experimental results.

We also validated the new code with regard to the longitudinal charge distribution. Figure 7 shows a complicated longitudinal double-peaked electron bunch shape after compression as it was directly measured with an interferometric method [13] along with simulation results obtained with Parmela and the two versions of Impact-T. 


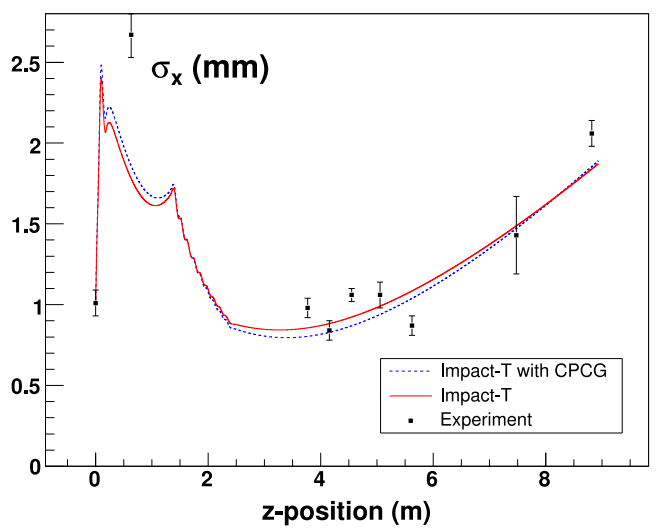

FIGURE 5. Beam radius at different distances from the cathode.

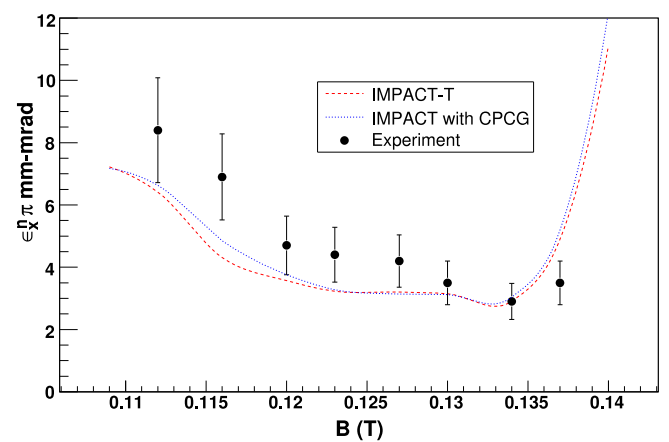

FIGURE 6. Measured and simulated transverse emittances as a function of the magnetic fields inside the emittance compensation solenoids.

\section{CODE APPLICATIONS}

We used our wavelet-based space charge algorithm to simulate and optimize the lattice of a new photoinjector which will be built at Jefferson Lab in cooperation with Advanced Energy Systems (AES) [14]. The main components of this photoinjector are a $6 \mathrm{MV} / \mathrm{m}$ dc gun, emittance compensation solenoids, three $750 \mathrm{MHz}$ accelerating cavities and a $3^{\text {rd }}$-harmonic cavity for longitudinal phase-space optimization.

The latest version of this photoinjector is designed to operate with a $1000 \mathrm{~mA}$ electron beam. The simulation results for the most important beam moments are shown in Fig. 8. The good agreement between Parmela and the two versions of Impact-T is an additional verification of the new wavelet-based code.

Simulation work also shows that there is a potential for halo formation when the AES/JLab photoinjector is operated under certain conditions (Fig. 9). We plan to extend our simulation studies to investigate fully what are the experimental conditions which could generate halo formation, and which could mitigate it. 


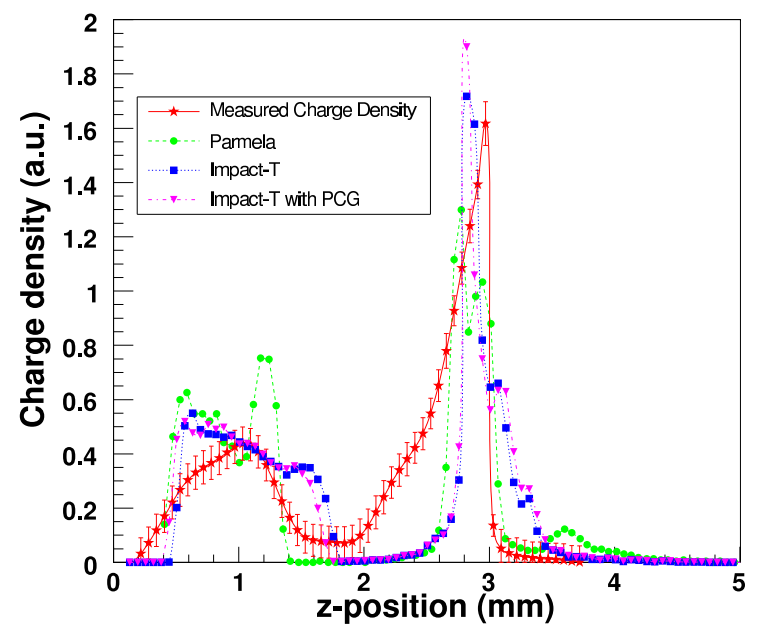

FIGURE 7. Longitudinal charge distribution for a double-peaked electron bunch shape after compression.
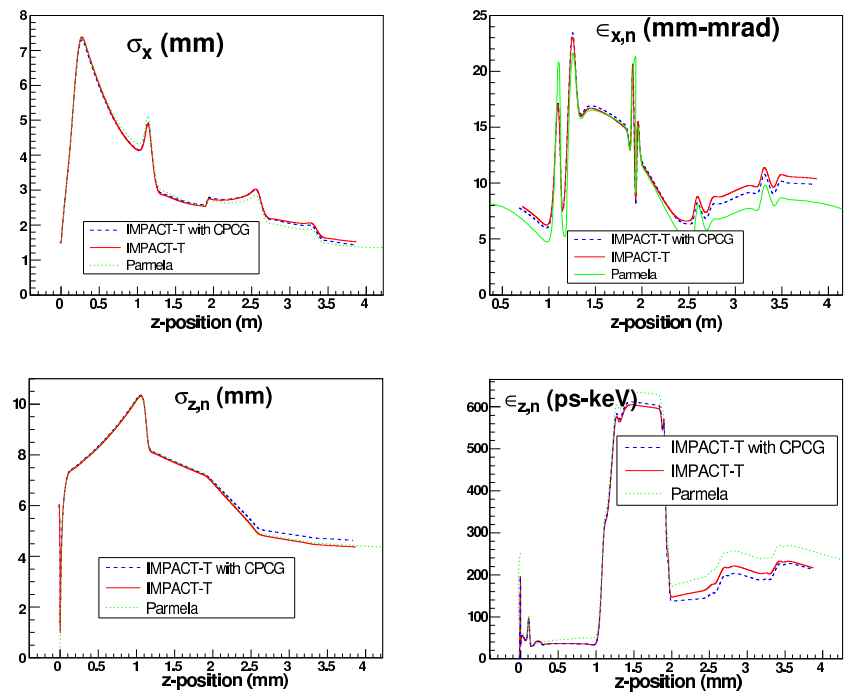

FIGURE 8. Beam moments for the $1000 \mathrm{~mA}$ version of AES/JLab photoinjector.

\section{CONCLUSIONS}

We developed a fully three-dimensional Poisson solver based on wavelet transforms. This new code also includes a denoising procedure and allows data compression for future code developments.

The machinery of the new Poisson solver is explained much more fully in Ref. [8]. To verify the new wavelet-based algorithm we compared the simulation results for beam moments and phase-space distributions with the standard Impact-T and Parmela codes. The agreement between codes is within $15 \%$. Code validation was done by comparing 

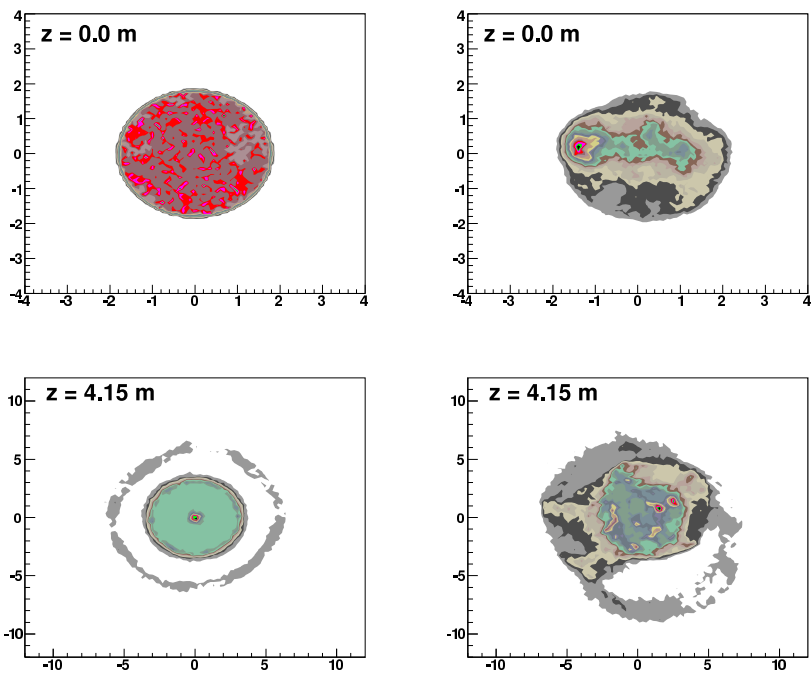

FIGURE 9. Transverse phase-space distributions for uniform (left) and non-uniform (right) initial distributions. $\mathrm{X}$ and $\mathrm{Y}$ are measured in milimiters.

the simulations with experimental measurements performed at the Fermilab/NICADD photoinjector. We used the new code to simulate the AES/JLab photoinjector, and we are presently using it to optimize its performance. This work was supported by DOE grant DE-FG02-04ER41323 and by Air Force contract FA9471-040C-0199.

\section{REFERENCES}

1. Linac Coherent Light Source (LCLS) Design Study Report, The LCLS Design Study Group, SLACR-521, (1998).

2. Tesla XFEL Technical Design Report, Desy 2002-167, (2002).

3. K. J. Kim, Nucl. Instrum. Methods Phys. Res., Sect. A 275, 201 (1989).

4. D. J. Gibson, F. V. Hartemann, E. C. Landahl, A. L. Troha, N. C. Lumann, G. P. Le Sage, and C. H. Ho, Phys. Rev. ST Accel. Beams 4, 090101 (2001).

5. Y. E. Sun, P. Piot, K. J. Kim, N. Barov, S. Lidia, J. Santucci, R. Tikhoplav, and J. Wennerberg, PRST-AB, 7, 123501 (2004).

6. L. Young, LANL report LA-UR-96-1835

7. J. Qiang, et al., Proceedings of 2005 IEEE Particle Accelerator Conference, Knoxville TN, p. 3316 (2005).

8. B. Terzič, I. V. Pogorelov, C. L. Bohn, submitted to J. Comp. Phys. (2006).

9. S. Goedecker, Wavelets and Their Applications (Presses polytechniques et universitaires romandes, Lausanne, 1998).

10. M. Wickerhauser, Adaptive Wavelet Analysis From Theory to Software (A K Peters, Wellesley, Massachusetts, 1994).

11. I. Daubechies, Ten Lectures on Wavelets (SIAM, Philadelphia, 1992).

12. Fermilab/NICADD website: http://nicadd.niu.edu/fnpl.

13. D. Mihalcea, C. L. Bohn, U. Happek, P. Piot, Longitudinal electron bunch diagnostics using coherent transition radiation, (will be published shortly) Phys. Rev. ST Accel. Beams, (2006).

14. A. Todd, et al., in Proceedings of 2003 IEEE Particle Accelerator Conference, Portland, OR, p. 977 (2003). 